\title{
Impact of Indoor-Outdoor Context on Crowdsourcing based Mobile Coverage Analysis
}

\author{
Mahesh K. Marina*, Valentin Radu*, Konstantinos Balampekos ${ }^{\dagger}$ \\ *The University of Edinburgh †Nokia Networks
}

\begin{abstract}
We consider the crowdsourcing based mobile cellular network measurement paradigm that is becoming increasingly popular. In particular, we aim to study the impact of user indoor/outdoor environment context at time of measurement. Focusing on signal strength as the measurement metric and using a real large crowdsourced measurement dataset for central London area along with estimated environment state (indoor or outdoor), we show that indoor-outdoor context has a significant impact, suggesting that conflating indoor and outdoor measurements can lead to unreliable results. We validate these observations using a set of diverse and controlled measurements with indoor/outdoor ground truth information. We also discuss some opportunities for future work (e.g., accurate and efficient context detection) relevant to crowdsourced mobile network measurement systems.
\end{abstract}

\section{CCS Concepts}

$\bullet$ Networks $\rightarrow$ Network measurement; Mobile networks;

\section{Keywords}

Crowdsourced mobile network measurement; indoor-outdoor environment context; cellular coverage

\section{INTRODUCTION}

Crowdsourcing [1-6] has recently emerged as a new approach for mobile cellular network measurement and analysis. It exploits smartphones (with built-in cellular network interface and location sensing capabilities) as measurement sensors and the natural mobility of people carrying them for cost-effective, continual and fine-grained spatio-temporal monitoring of mobile networks. The crowdsourcing approach has several advantages over existing approaches like drive or classroom use is granted without fee provided that copies are not made or distributed for profit or commercial advantage and that copies bear this notice and the full citation on the first page. Copyrights for components of this work owned by others than the author(s) must be honored. Abstracting with credit is permitted. To copy otherwise, or republish, to post on servers or to redistribute to lists, requires prior specific permission and/or a fee. Request permissions from permissions@acm.org.

AllThingsCellular'15, August 17-21 2015, London, United Kingdom

(C) 2015 Copyright held by the owner/author(s). Publication rights licensed to ACM. ISBN 978-1-4503-3538-6/15/08. . \$15.00

DOI: http://dx.doi.org/10.1145/2785971.2785976 testing (e.g., [7]), coverage modelling (e.g., [8]) and networkside passive analysis (e.g., [9]). It captures reality better than the coverage modelling approach; less expensive than the drive testing approach; and unlike the network-based passive monitoring approach, it allows direct measurement at user side including context. Also measurements with the crowdsourcing approach reflect user perceived mobile performance as they are obtained from real end-user devices. The foregoing discussion suggests that the crowdsourcing approach will likely be an integral part of a broader approach to meet the emerging mobile network measurement and monitoring needs.

However the crowdsourcing approach also presents several challenges some of which have been discussed in [10]; one of the challenges mentioned concerns device and environment context and is the focus of this paper: ".. it is not always clear where the device is located when the test is made (so it could be indoors or outdoors, in a bag or in the user's hand) ..." Gember et al. [4] have in fact partially characterized the impact of some of the relevant contextual factors, especially device position (phone in hand or not), and show that such factors have a significant impact on measurement results. For example, [4] reports that moving the phone from hand to pocket can cause up to $79 \%$ difference in measured throughput.

In this paper, we complement the previous work in [4] by showing that whether a mobile user participating in a crowdsourced measurement system is indoor or outdoor at the time of measurement matters significantly. This is an environment related aspect of user context that has not received much attention till date except for brief mentions such as in [5], where the authors state that not knowing whether a measurement was taken indoors or outdoors could lead to bias in measurement results. Also, existing mobile crowdsourcing systems (e.g., [1,2]) do not feature the indoor-outdoor detection capability.

To study the indoor-outdoor impact, we focus on signal strength (RSSI) as the measurement metric driven by three reasons. Firstly, coverage or signal strength is not only an intuitive metric for users but also the primary metric targeted by operators and regulators; this is clearly stated by the authors of [11] "Without exaggeration, we can say that coverage is the most important and the highest-priority target that has to be achieved by cellular operators." and is also evident 


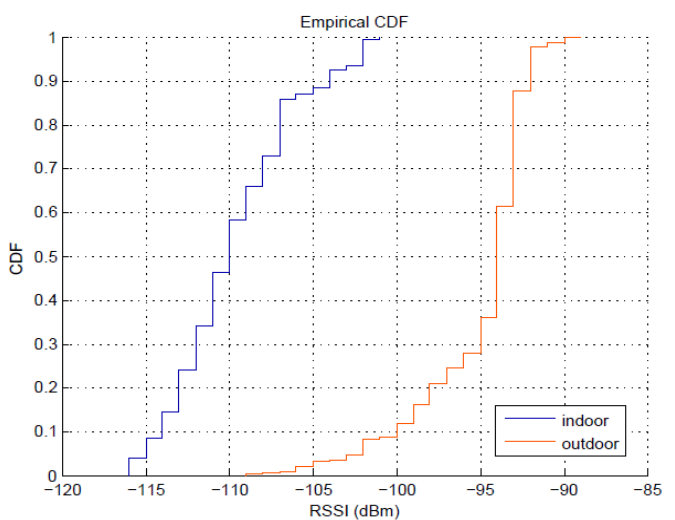

Figure 1: CDF of indoor and outdoor RSSI values for a specific cell sector and operator combination (OpenSignal dataset).

from [8]. Secondly, signal strength is recently shown to correlate well with throughput and mobile device battery energy drain $[6,12,13]$. Finally, unlike other performance metrics like throughput that may require active measurement, signal strength can be measured passively with little or no impact of device battery consumption; for this reason, it is also the most widely supported metric across all existing crowdsourced mobile network measurement systems.

In particular, we highlight the downside of conflating indoor and outdoor measurements. Such conflation of measurements from different environments and contexts is quite likely to happen in practice because it is typical to aggregate nearby measurements into coarser geographic units such as grid squares or postcodes. It is easy to find examples from real-world practice and research literature where aggregation of measurements over space is done. Ofcom's mobile coverage analysis [8] aggregates coverage predictions into postcodes and 200mx200m grid squares. WiScape [3] partitions the world into zones each around $0.2 \mathrm{sq}$. $\mathrm{km}$. each. Online coverage checkers on websites of mobile operators typically indicate coverage at the level of postcodes (e.g., [14]).

Using a large crowdsourced measurement dataset, consisting of nearly 8 million measurements spanning over 3 years, from OpenSignal [1] for central London, we characterize the differences in received mobile signal characteristics between indoors and outdoors and more crucially, highlight the risk of conflating indoor and outdoor measurements (§2). As existing crowdsourced measurement systems do not differentiate between indoor and outdoor measurements and spatial aggregation of measurements is common, conflation of diverse measurements can thus lead to erroneous conclusions - coverage outdoors is likely to be underestimated, whereas indoor coverage may be overestimated. Imprecision or uncertainty concerning location of a measurement can compound this problem. We validate the above mentioned risk using a diverse set of controlled measurements with indoor/outdoor ground truth information (§3). We also discuss related aspects and issues for future research in $\S 4$.

\section{ANALYSIS WITH A CROWDSOURCED MEASUREMENT DATASET}

In this section, we study the impact of conflating indoor and outdoor mobile measurements using a large crowdsourced dataset from OpenSignal [1].

\subsection{Dataset}

Our study is based on OpenSignal dataset for central London. These measurements are collected via OpenSignal Android app and span an area around 58 square kilometers with 15980 post codes. The dataset includes signal strength measurements from $3 \mathrm{G}$ mobile networks (UMTS, HSDPA, HSUPA, HSPA+) of all four major mobile telecom operators in the UK (Vodafone, O2, Everything Everywhere and Three) and overall contains 42976 distinct cell sectors with measurements. Measurements span a period of 3 years and 4 months dating back from July 2013. There are around 7.75 million distinct measurements in the dataset.

\subsection{Mechanism for Differentiating Indoor and Outdoor Measurements}

As noted at the outset, OpenSignal and for that matter all other existing crowdsourcing based mobile network measurement systems do not feature the indoor-outdoor detection capability. The only source of relevant context information available with each measurement record is the location information obtained at the time of measurement via the Android Location API. Therefore we choose to rely on a GPS based method used previously in [15] (and mentioned in [4]) to infer whether a measurement was collected while indoors or outdoors. The GPS based method essentially treats all measurements with a location obtained via GPS fix as outdoor measurements, and the rest of the measurements with network based location (via Wi-Fi or mobile network) are categorized as indoor measurements. This method is simplistic and as we show in [16], it provides an accuracy in the region of $75-80 \%$ but in our view it is the only reasonable way for ex post facto determination of indoor-outdoor context with a dataset of this kind. As we will show subsequently in Section 3, results obtained using the GPS method qualitatively agree with those collected in a controlled manner with actual ground truth indoor/outdoor context information. Relying on GPS for indoor-outdoor detection, however, has a big downside in terms of device energy consumption, as discussed later.

\subsection{Results}

In order to appreciate the potential differences in received signal strength characteristics between indoor and outdoor environments, let us start by focusing on the distribution of "indoor" and "outdoor" RSSI values corresponding to a specific cell sector of a mobile network in operation. Results are shown in Fig. 1. Separation of indoor and outdoor measurements is done using the GPS based method described above. The example combination of operator and cell sector is chosen arbitrarily; it consists of 555 measurements in total, and at least 200 indoor and outdoor measurements. Note that 


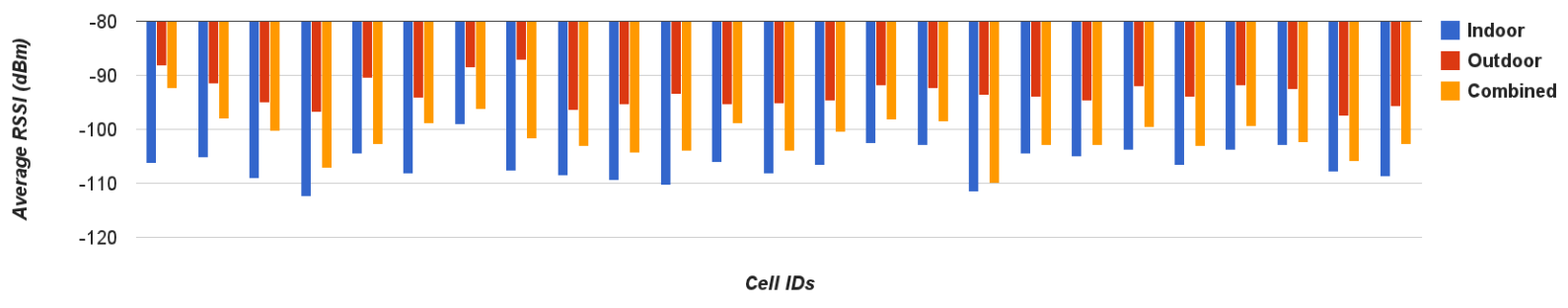

Figure 2: Differences in indoor and outdoor average RSSI values for a selection of 25 cell sectors.

cell sector offers the right granularity for our analysis - any finer unit will limit the number of measurements to make statistically valid conclusions, whereas more coarser units will introduce other confounding factors. Previous work [4] has also observed that differences in mobile performance measurements can be largely explained as due to changes in cell sector.

From Fig. 1, we can observe that indoor and outdoor RSSI values can be clearly demarcated with median RSSI between the two cases differing by more than $15 \mathrm{dBm}$. This is along the lines of results from measurement studies on attenuation of signals propagating into buildings [17, 18]. To put these results into perspective, consider that Ofcom (the UK's telecommunications regulator) in [19] has defined $>-91.7 \mathrm{dBm}$ and $<-105.5 \mathrm{dBm}$ as signal strength thresholds to reflect excellent and poor $3 \mathrm{G}$ coverage, respectively. Outdoor RSSI values shown in Fig. 1 are near the "excellent" coverage threshold whereas most of the indoor RSSI values are lower than the threshold for "poor" coverage.

Having looked at a specific case, let us now obtain a more general picture on the differences between indoor and outdoor contexts from a signal strength viewpoint. Fig. 2 shows a selection of 25 cell sectors from those with at least 150 measurements each for indoor and outdoor (there are 661 such cell sectors in the dataset). We can observe that the average signal strength between indoor and outdoor differs by at least $10 \mathrm{~dB}$ for each of the 25 chosen sectors. When indoor and outdoor measurements are not explicitly differentiated, the result is conflation of measurements with quite different characteristics. The result of such conflation is shown by the orange colored "Combined" bars in Fig. 2. The combined case is biased towards the indoor because in most of the cells there are many more indoor measurements than those from outdoors, consistent with the fact that mobile users spend $80 \%$ of their time indoors [20].

Fig. 3 shows the CDF of differences in average RSSI between indoors and outdoors across all 661 cell sectors with a minimum of 150 measurements each for indoor and outdoor. Even at this level, we see that in nearly a quarter of the cases outdoor signal strength values are substantially greater (by $5 \mathrm{~dB}$ or more) than those from indoors, indicating that indoor-outdoor context is clearly an important factor that needs to be accounted for when analyzing crowdsourced mobile measurements. Concerning the other extreme where indoor RSSI values exceed those outdoors, this is not sur- prising when we consider that signal strength can be worse even while outdoors when near cell edge or as a result of signal reception subject to shadowing.

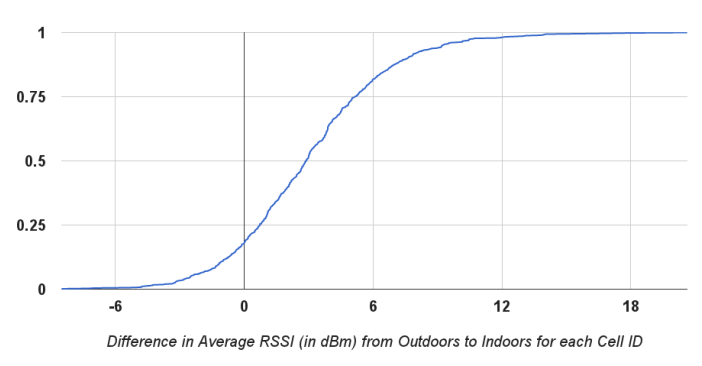

Figure 3: Distribution of differences in indoor and outdoor average RSSI values across all 661 cell sectors with at least 150 measurements each for indoors and outdoors.

By way of summarizing the consequence of the above analysis results with the OpenSignal dataset, we now illustrate the risk of conflating measurements from indoor and outdoor environments. For this, we consider two representative postcodes in central London with at least 1500 measurements per operator: SE1 0XN (SE11 5HY) with a total of 2967 (1684) measurements for Operator 1 (2) of which 2836 (1388) were estimated to be indoor (via the GPS method). We color code these postcode areas on a map using the Ofcom defined thresholds mentioned above: $>-91.7 \mathrm{dBm}$ as Excellent and $<-105.5 \mathrm{dBm}$ as Poor; other intermediate levels with thresholds evenly distributed between the extremes, specifically: $-105.5 \mathrm{dBm}<$ Fair $<-100.9 \mathrm{dBm}<$ Average $<-96.3 \mathrm{dBm}<$ Good $<-91.7 \mathrm{dBm}$.

Fig. 4 (a) and (d) shows the default case of conflating indoor and outdoor measurements for the two chosen postcodes. These results are clearly different from the corresponding indoor and outdoor coverage maps, respectively Fig. 4 (b, e) and (c, f). Combined results paint a mixed picture biased towards the indoor results for reasons mentioned above but it is clear that considering indoor and outdoor measurements separately would lead to a more reliable analysis. 


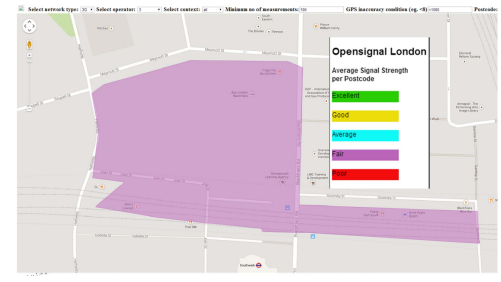

(a) SE1 OXN, combined

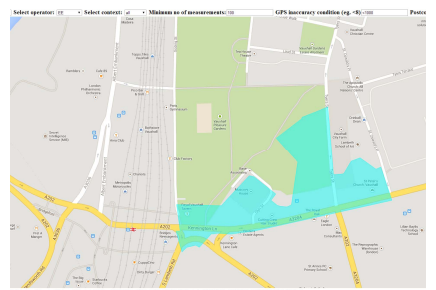

(d) SE11 5HY, combined

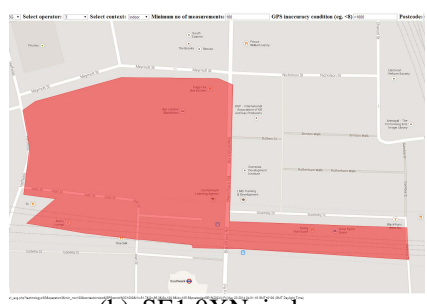

(b) SE1 OXN, indoor

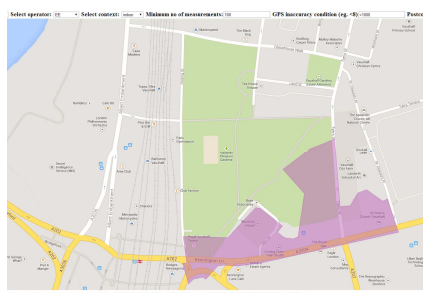

(e) SE11 5HY, indoor

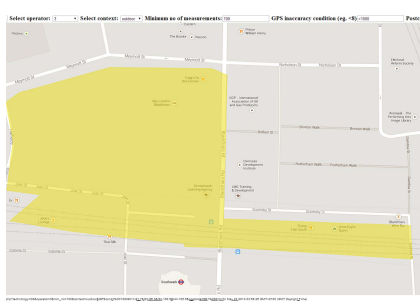

(c) SE1 OXN, outdoor

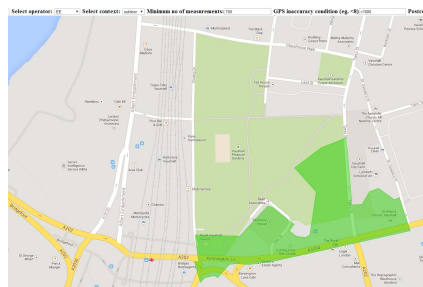

(f) SE11 5HY, outdoor

Figure 4: Risk of conflating indoor and outdoor measurements illustrated using measurements for two representative postcodes in central London.

\section{VALIDATION WITH CONTROLLED MEASUREMENTS}

The previous section studied the indoor-outdoor context impact on mobile network measurement using a crowdsourced dataset with measurements collected in the wild along with ex post facto inference of indoor-outdoor context information via the GPS method. In this section, we confirm our observations from the earlier study with a set of controlled measurements.

\subsection{Data Collection}

For this validation purpose, we collected signal strength measurements in Edinburgh using a custom Android app similar to the OpenSignal app but with an interface for volunteers assisting with the data collection to manually input indoor/outdoor ground truth information. Each measurement record with this app therefore consists of cellular network related information (time, location, mobile network code, network type, location area code, cell ID, signal strength, etc.) annotated with true indoor/outdoor context information. Our measurement data spans several different urban settings including office, shopping areas and residential locations, both indoors and outdoors. It consists of more than 5000 measurement records across 18 distinct cell sectors.

\subsection{Results}

Our presentation of results in this section mimics that in section 2, i.e., starting with specific examples and progressing to more general results. We start by looking at the indoor and outdoor RSSI distribution within two specific cells

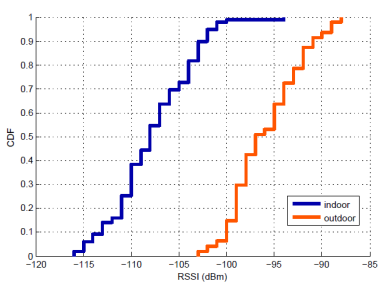

(a) Office

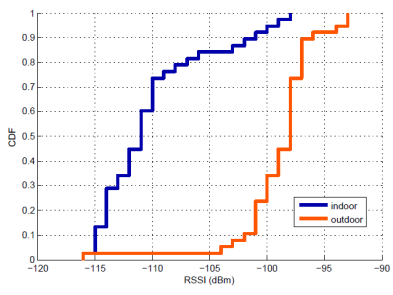

(b) Shop
Figure 5: CDF of indoor and outdoor RSSI values within a cell in two different settings with controlled measurements: (a) office and (b) shop.

corresponding to two different measurement settings: office and shop (Fig. 5). RSSI distributions for indoor and outdoor contexts in both settings are qualitatively similar to those reported earlier in Fig. 1.

Let us now look at the average RSSI differences between indoor and outdoor contexts in each of the 18 different cell sectors in the controlled dataset. Results, shown in Fig. 6 (a), are qualitatively similar to those seen earlier in Fig. 2, showing the effect of conflating indoor and outdoor measurements. They also include a case where outdoor signal strength is lower than indoor, which happens in reality near cell edge or due to shadowing effects and again something encountered previously with the OpenSignal dataset (see Fig. 3). Note that indoor results are actually composed of measurements at different floors and parts inside the buildings whereas the outdoor results are made up of measurements taken on different sides of buildings. Based on this, 


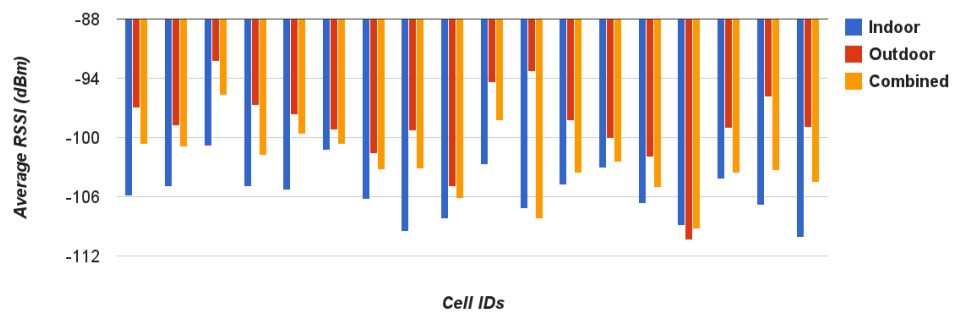

(a)

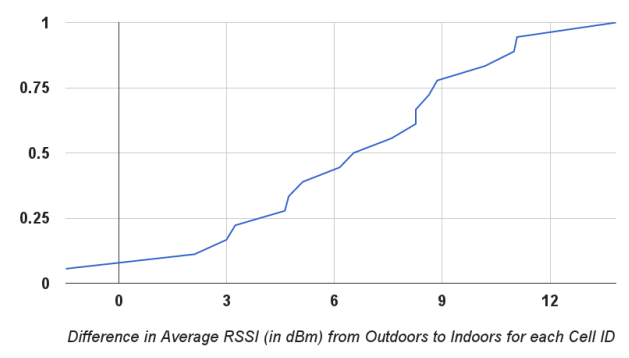

(b)

Figure 6: (a) Differences in indoor and outdoor average RSSI values for each cell sector in the controlled measurement dataset. (b) Distribution of differences in indoor and outdoor average RSSI values across all cell sectors in the controlled measurement dataset.

we can observe that conflating indoor or outdoor measurements separately is less of a problem compared to conflating indoor and outdoor measurements. The CDF of indoor and outdoor average RSSI differences per cell in the controlled dataset is shown in Fig. 6 (b). While qualitatively similar to the corresponding result with the OpenSignal dataset, it also shows more pronounced effect of indoor-outdoor context outdoor signal strength values are greater than indoor values by $5 \mathrm{~dB}$ in nearly $60 \%$ of the cases though this could just be an artifact of relatively smaller number of cell sectors in the dataset.

\section{DISCUSSION}

\subsection{Other Contextual Factors}

Having looked more closely at the impact of indoor-outdoor context on mobile performance, it is useful to briefly discuss the broader question concerning the impact of different contextual factors. While WiScape [3] has considered spatio-temporal variations in performance, the work in [4] is the closest related work in the literature addressing this question. Specifically, Gember et al. in [4] have studied the impact of device position (e.g., hand, desk, bag, pocket), movement (e.g., stationary, walking, driving) and location; they conclude that all these different contextual factors affect performance substantially so need to be taken into account. It is worth noting though that the focus of the work in [4] is on performance when the user is actively interacting with their device, which they refer to as "in-context" measurement. However recent work [21,22] has highlighted the significant wasteful device energy consumption and signaling traffic due to background/idle communication. For example, Huang et al. [21] report that $58.5 \%$ of the device radio energy consumption is caused by off-screen traffic. Recognizing the importance of monitoring at both active and idle/background periods, our analysis of indoor-outdoor context impact spans both periods.

\subsection{Accurate and Efficient Context Detec- tion}

There is a sort of irony with crowdsourced mobile net- work measurement approach when it comes to context. On one hand, the crowdsourcing approach is better placed to account for user-side contextual factors. On the flip side, accurate and energy-efficient context detection is a major challenge for the crowdsourcing approach. The latter is evident when we consider the issue of localizing measurements. Existing systems take one of two extremes. At one extreme, exemplified by OpenSignal [1], a precise location for each measurement is obtained by relying on GPS when possible and using network based location as a fallback; this is however an energy hungry strategy as GPS is the among the most energy consuming sensors on a mobile device [16]. Systems like MobiPerf [2] take the other extreme of just using network based location always; while relatively energy-efficient this can lead to quite high imprecision (in the order of $100 \mathrm{~s}$ of meters) in the location of measurements.

Turning attention to the indoor-outdoor context detection, the key focus of this paper, we mentioned earlier and also shown experimentally in [16] that GPS based method only gives an accuracy around $75-80 \%$. But a bigger limitation of this method is that it is quite expensive from an energy consumption viewpoint as it relies on GPS. The other existing approach for indoor-outdoor detection [23] while energyefficient is not reliable across different environments as it relies on fixed thresholds for sensor values (light, magnetic and cell signal). In [16], we have developed a semi-supervised learning based solution that is robust across environments while at the same time accurate $(>90 \%)$ and energy-efficient. Generalizing such a solution to address the accurate, energyefficient detection of other contexts (location, etc.) and realizing a context-aware, energy-efficient crowdsourced mobile network measurement system is not fully addressed, and is the focus of our on-going work.

\subsection{Impact on Performance}

As our analysis focused solely on signal strength, a relevant question is the implication of our observations on the impact of indoor-outdoor context on performance metrics such as throughput. We can address this question by referring to the recent work of Nikravesh et al. [6] that studies the impact of signal strength on performance metrics using 
MobiPerf [2]. Specifically, [6] reports that, except at very high signal strengths, performance (in terms of throughput, latency and packet loss) depends on signal strength in an expected way - lower the signal strength, lower the throughput and higher the latency and loss. While signal strength is a good proxy for performance in lightly loaded conditions and is also useful for inferring maximum achievable throughput, it may not be a reliable indicator during peak traffic periods and highly loaded network conditions.

Note that our analysis was based on signal strength measurements of $3 \mathrm{G}$ networks which in the UK operate solely in 1900/2100 MHz band. Because of the frequency dependence of outdoor and outdoor-to-indoor signal propagation $[17,18]$, the impact of indoor-outdoor context on coverage analysis will be different at different frequency bands; further exploration of this is an issue for future work. Another relevant point concerns the growing deployment of femto cells inside homes and offices, which mean that the cell that a device associates with indoors might differ from that while outdoors. This however does not reduce the importance of estimating macro/small cell signal reception inside buildings which would be key to ensuring blanket coverage and meeting the requirements of emergency (E-911 type) services.

\section{CONCLUSIONS}

In this paper, we have further highlighted the importance of context awareness in crowdsourcing based mobile cellular network measurement systems. Specifically, we have studied the impact of user environment (indoor or outdoor) at the time of measurement. Our analysis, focusing on signal strength measurements and using a combination of large crowdsourced dataset and controlled measurements, revealed that indoor-outdoor user environment context matters significantly and therefore conflation of indoor and outdoor measurements should be avoided.

\section{ACKNOWLEDGMENTS}

We thank OpenSignal for providing access to the dataset used in this study.

\section{REFERENCES}

[1] Open Signal Inc. http://opensignal.com/.

[2] MobiPerf. http://www.mobiperf.com/.

[3] S. Sen, J. Yoon, J. Hare, J. Ormont, and S. Banerjee. Can they hear me now?: A Case for a Client-Assisted Approach to Monitoring Wide-Area Wireless Networks. In Proc. ACM Internet Measurement Conference (IMC), 2011.

[4] A. Gember, A. Akella, J. Pang, A. Varshavsky, and R. Caceres. Obtaining In-Context Measurements of Cellular Network Performance. In Proc. ACM Internet Measurement Conference (IMC), 2012.

[5] J. Sommers and P. Barford. Cell vs. WiFi: On the Performance of Metro Area Mobile Connections. In Proc. ACM Internet Measurement Conference (IMC), 2012.

[6] A. Nikravesh, D. R. Choffnes, E. Katz-Bassett, Z. M. Mao, and M. Welsh. Mobile Network Performance from User Devices: A Longitudinal, Multidimensional Analysis. In Proc. Passive and Active Measurement (PAM) Conference, 2014.
[7] C. N. Pitas, A. D. Panagopoulos, and P. Constantinou. Speech and Video Telephony Quality Characterization and Prediction of Live Contemporary Mobile Communication Networks. Wireless Personal Communications, 69(1):153-174, Mar 2012.

[8] Ofcom. Infrastructure Report 2014: Interactive Maps and Infographics. http://infrastructure.ofcom.org.uk/.

[9] A. Gerber, J. Pang, O. Spatscheck, and S. Venkataraman. Speed Testing without Speed Tests: Estimating Achievable Download Speed from Passive Measurements. In Proc. ACM Internet Measurement Conference (IMC), 2010.

[10] Ofcom. Measuring Mobile Voice and Data Quality of Experience. http://stakeholders.ofcom.org.uk/consultations/ mobile-voice-data-experience/, Jan 2013.

[11] A. Galindo-Serrano, B. Sayrac, S. Ben Jemaa, J. Riihijarvi, and P. Mahonen. Automated Coverage Hole Detection for Cellular Networks Using Radio Environment Maps. In Proc. 9th International Workshop on Wireless Network Measurements (WiNMee), 2013.

[12] C. Cheng and P. Hsiu. Extend Your Journey: Considering Signal Strength and Fluctuation in Location-Based Applications. IEEE/ACM Transactions on Networking, 22, Feb 2014.

[13] N. Ding, D. Wagner, X. Chen, A. Pathak, Y. Charlie Hu, and A. Rice. Characterizing and Modeling the Impact of Wireless Signal Strength on Smartphone Battery Drain. In Proc. ACM SIGMETRICS, 2013.

[14] Ofcom. Mobile Network Coverage Checkers. http://ask.ofcom.org.uk/help/telephone/mobilecoverage.

[15] L. Ravindranath, C. Newport, H. Balakrishnan, and S. Madden. Improving Wireless Network Performance Using Sensor Hints. In Proc. USENIX NSDI, 2011.

[16] V. Radu, P. Katsikouli, R. Sarkar, and M. K. Marina. A Semi-Supervised Learning Approach for Robust Indoor-Outdoor Detection with Smartphones. In Proc. ACM SenSys, 2014.

[17] D. M. Rose and T. Kurner. Outdoor-to-Indoor Propagation Accurate Measuring and Modelling of Indoor Environments At 900 and $1800 \mathrm{MHz}$. In Proc. 6th European Conference on Antennas and Propagation (EUCAP'12), 2012.

[18] I. Rodriguez et al. Radio Propagation into Modern Buildings: Attenuation Measurements in the Range from $800 \mathrm{MHz}$ to 18 GHz. In Proc. 80th IEEE Vehicular Technology Conference (VTC Fall'14), 2014.

[19] Ofcom. Mobile Coverage Information for Consumers. http://stakeholders.ofcom.org.uk/binaries/research/ telecoms-research/not-spots/mobile-coverage/ mobile-coverage-information.pdf, Aug 2011.

[20] N. Klepeis et al. The National Human Activity Pattern Survey (NHAPS): A Resource for Assessing Exposure to Environmental Pollutants. Journal of Exposure Analysis and Environmental Epidemiology, 11:231-252, 2001.

[21] J. Huang, F. Qian, Z. M. Mao, S. Sen, and O. Spatscheck. Screen-Off Traffic Characterization and Optimization in 3G/4G Networks. In Proc. ACM Internet Measurement Conference (IMC), 2012.

[22] A. Aucinas et al. Staying Online While Mobile: The Hidden Costs. In Proc. ACM CoNEXT, 2013.

[23] P. Zhou, Y. Zheng, Z. Li, M. Li, and G. Shen. IODetector: A Generic Service for Indoor Outdoor Detection. In Proc. ACM SenSys, 2012. 